\title{
Does maternal smoking in pregnancy explain the differences in the body composition trajectory between breastfed and formula-fed infants?
}

\author{
Shao J. Zhou ${ }^{1 *}$, Karen Hawke ${ }^{2}$, Carmel T. Collins ${ }^{2,3}$, Robert A. Gibson ${ }^{1,2}$ and Maria Makrides ${ }^{2,3}$ \\ ${ }^{1}$ School of Agriculture, Food and Wine, The University of Adelaide, Waite Campus, Urrbrae, SA 5064, Australia \\ ${ }^{2}$ South Australian Health and Medical Research Institute, Adelaide, SA 5000, Australia \\ ${ }^{3}$ Discipline of Paediatrics, Adelaide Medical School, The University of Adelaide, Adelaide, SA 5005, Australia \\ (Submitted 14 May 2019 - Final revision received 23 September 2019 - Accepted 29 October 2019 - First published online 8 November 2019)
}

\section{Abstract}

Growth patterns are known to differ between breastfed and formula-fed infants, but little is known about the relative impact of maternal smoking in pregnancy $v$. feeding mode on growth trajectory in infancy. We conducted a secondary analysis of a trial, the Tolerance of Infant Goat Milk Formula and Growth Assessment trial involving 290 healthy infants, to examine whether smoking in pregnancy modified the association between feeding mode and body composition of infants. Fat mass (FM) and fat-free mass (FFM) were estimated at 1, 2, 3, 4, 6 and 12 months of age using bioimpedance spectroscopy. Formula-fed infants ( $n$ 190) had a higher mean FFM at 4 months (mean difference (MD) $160 \mathrm{~g}$, $95 \% \mathrm{CI}$ $50 \cdot 4,269.5 \mathrm{~g}, P<0.05)$ ) and 6 months (MD $179 \mathrm{~g}, 95 \% \mathrm{CI} 41.5,316.9 \mathrm{~g}, P<0.05)$ compared with the breastfed infants ( $n 100)$. Sub-group analysis of breastfed $v$. formula-fed infants by maternal smoking status in pregnancy showed that there were no differences in the FM and FFM between the breastfed and formula-fed infants whose mothers did not smoke in pregnancy. Formula-fed infants whose mothers smoked in pregnancy were smaller at birth and had a lower FM\% and higher FFM\% at 1 month compared with infants of non-smoking mothers regardless of feeding mode, but the differences were not significant at other time points. Adequately powered prospective studies with an appropriate design are warranted to better understand the relative impact of maternal smoking, feeding practice and the growth trajectory of infants.

Key words: Infants: Growth: Body composition: Breast-feeding: Formula feeding: Maternal smoking

Growth patterns are known to differ between breastfed and formula-fed infants in the first year of life. Formula-fed infants generally gain more weight and are heavier by 12 months of age $^{(1,2)}$, which has been attributed to the differences in nutritional composition between breast milk and infant formula particularly the higher content of protein in infant formula ${ }^{(3)}$. The differential growth pattern between breastfed and formula-fed infants has been interpreted by some to mean that formula-fed infants are fatter when compared with breastfed infants $^{(4)}$, and this is thought to contribute to the higher risk of developing overweight and obesity in formula-fed infants over the life course. A recent systematic review and meta-analysis ${ }^{(2)}$ showed that formula-fed infants had a lower percentage of fat mass (FM) at 3.5 months and at 6 months compared with breastfed infants, but the percentage of FM was less consistent for the second 6 months of the infancy (a trend for a lower percentage of FM at around 8 months and higher percentage at 12 months). This may partly be due to a small number of eligible studies included in the review and the different definitions used to define breastfed and formula-fed groups among studies. Only three of the fifteen included studies, with relatively small sample sizes (46-100 infants), had at least one body composition measurement in each of the first and second 6 months of the infancy $^{(2)}$.

There is some evidence suggesting that breast-feeding for longer than $6^{(5,6)}$ or 12 months ${ }^{(7)}$ is associated with lower FM in early childhood. However, evidence for the association between breast-feeding in infancy and lower risk of overweight and obesity over the life course has been inconclusive ${ }^{(8)}$. This is perhaps not surprising as there is increasing recognition that while early nutrition plays a key role, there are other environmental factors that may also influence the growth pattern and risk of developing obesity later in life. One of the recognised factors that influence growth in early life is maternal smoking during pregnancy, which has been linked to fetal growth restriction, lower birth weight ${ }^{(9,10)}$ and lower FM and fat-free mass (FFM)

Abbreviations: BIS, bioimpedance spectroscopy; FM, fat mass; FFM, fat-free mass; MD, mean difference; TIGGA, Tolerance of Infant Goat Milk Formula and Growth Assessment.

* Corresponding author: Shao J. Zhou, fax +61888313 7135, email jo.zhou@adelaide.edu.au 
at birth ${ }^{(11-13)}$ but higher FFM at 5 months ${ }^{(12)}$. Being born small with rapid catch-up growth in early infancy has been shown to increase the risk of developing obesity later in life ${ }^{(14,15)}$. Despite this, few studies have considered the impact of maternal smoking in pregnancy when examining the relationship between feeding mode and growth pattern or body composition in infancy.

The aims of the study were to examine the relationship between feeding mode (breastfed $v$. formula-fed) and body composition trajectory of infants in the first 12 months of life and to examine whether the relationship between feeding mode and infant body composition differed by maternal smoking status in pregnancy.

\section{Methods}

\section{Study population}

The present study is an exploratory analysis of data collected in the Tolerance of Infant Goat Milk Formula and Growth Assessment (TIGGA) study, which is a randomised controlled trial comparing the growth and nutritional status of infants-fed goat-milk $v$. cow-milk infant formula. The TIGGA trial was registered at the Australian New Zealand Clinical Trials Registry (ACTRN12608000047392, http://www.anzctr.org.au/ Default.aspx). The detailed study design of the TIGGA trial has been reported elsewhere ${ }^{(16,17)}$. Briefly, 301 healthy full-term infants (200 formula-fed and 101 breastfed) up to 2 weeks of age were recruited from the postnatal ward in three hospitals in Adelaide between April 2008 and April 2009. Infants were eligible to participate in the TIGGA study if they were born full term (gestation 37-42 weeks) and a birth weight between 2.5 and $4.75 \mathrm{~kg}$. In addition, infants had to be exclusively fed infant formula within 2 weeks of birth (for the formula-fed cohort) or the mother planned to exclusively breastfeed for at least the first 4 months of life (for the breastfed cohort). Infants were excluded if they were from multiple births or had severe congenital or metabolic disease likely to affect feeding or growth. Formula-fed infants were randomly assigned to receive either goat-milk infant formula or cow-milk infant formula from enrolment until at least 4 months of age, and thereafter in conjunction with other complementary foods until 12 months of age ${ }^{(17)}$. For the breastfed comparison group, mothers were encouraged to exclusively breastfeed for 4-6 months of age ${ }^{(17)}$. Compliance with exclusive formula feeding or breast-feeding, defined as the infant receiving only formula (for formula-fed) or breast milk (for breast-f eeding) and no other liquids or solids with the exception of drops or syrups consisting of vitamins, mineral supplements or medicines ${ }^{(18)}$, from enrolment to 4 months of age was observed in $67 \%$ of formula-fed infants and $75 \%$ of breastfed infants ${ }^{(17)}$. The time to introduce solids between 4 and 6 months of age was at the discretion of each individual family for both the formula-fed and breastfed infants.

The present study was conducted according to the guidelines laid down in the Declaration of Helsinki, and all procedures involving human subjects/patients were approved by the Human Research Ethics Committees of the Women's and Children's Health Network, Flinders Medical Centre and Lyell
McEwen Hospital. Written informed consent was obtained from all participating families. The procedures followed were in accordance with the ethical standards of the responsible Human Research Ethics Committees.

\section{Outcome assessments}

The primary outcome measures were FM (g) and FM\%, and FFM (g) and FFM\%. The secondary outcome measures included weight, length and head circumference and their $z$-scores. FM and $\mathrm{FM} \%$, and FFM and FFM\% were measured at 1, 2, 3, 4, 6 and 12 months of age. FFM and FM were estimated by bioimpedance spectroscopy (BIS), which has been validated for measuring body composition in infancy ${ }^{(19)}$. The protocol followed has been described in detail previously ${ }^{(20)}$. In brief, BIS measurements were performed using an ImpediMed SFB7 (ImpediMed), which is a single channel, tetra-polar BIS device that measures impedance, resistance and reactance at 256 logarithmically spaced frequencies between 4 and $1000 \mathrm{kHz}$. A minimum of ten impedance readings were taken at 1-s intervals using the automated repeat reading mode. Impedance measurement was continued until a valid set of measurements were obtained, which was indicated by minimal scattering of data points and the presence of a well-fitted Cole plot ${ }^{(20)}$. Weight, length and head circumference of the infants were also measured at birth, enrolment and 1, 2, 3, 4, 6 and 12 months of age in the TIGGA study using standard methods ${ }^{(17)}$. $z$-Scores for weight, length and head circumference were calculated using WHO Child Growth Standards (http:// www.who.int/childgrowth/en/).

\section{Other assessments}

A structured interview questionnaire was used to assess maternal characteristics including age, parity, weight, height, education level and smoking during pregnancy (yes or no) at study entry, and feeding practice during the study period. Infant sex and age at enrolment were also recorded.

\section{Statistical analysis}

FFM (g) was calculated using the $W+S+L^{2} / R_{0}$ equations by Lingwood et $a l .{ }^{(19)}$, where $R_{0}$ is the resistance at zero frequency, $W$ is the weight in $\mathrm{kg}, S$ is the sex ( $1=$ male, $2=$ female) and $L$ is the length in $\mathrm{cm}$ at the time of BIS measurement. The equation for 6 weeks $\left(1.169+0.568 W-0.128 S+0.032 L^{2} / R_{0}\right)$ was used for FFM at 1 and 2 months; the equation for 3 months $\left(1 \cdot 315+0.449 W-0 \cdot 169 S+0 \cdot 153 L^{2} / R_{0}\right)$ was used for FFM at 3 months; and the equation for 4.5 months $(1.909+0.280 \mathrm{~W}-$ $0 \cdot 279 S+0 \cdot 305 L^{2} / R_{0}$ ) was used for FFM at 4,6 and 12 months. FM was then calculated as (weight $(\mathrm{g})$ - FFM), FM percentage as (FM/weight) $\times 100$ and FFM percentage as (FFM/ weight) $\times 100$.

To address our aims, we first tested whether body composition differed between the goat-milk and cow-milk formula-fed groups. As there were no differences in the anthropometric measures ${ }^{(17)}$ and body composition (see online Supplementary Table S1) between the two formula-fed groups, data for the two formulafed groups were combined to form the combined formula-fed group for comparison with the breastfed group. To examine 
whether the association between feeding mode and body composition differed by maternal smoking status in pregnancy, the infants were further divided into four groups according to feeding mode and maternal smoking status in pregnancy as follows: (1) breastfed non-smoking group: breastfed infants whose mothers did not smoke in pregnancy; (2) breastfed smoking group: breastfed infants whose mothers smoked in pregnancy; (3) formula-fed non-smoking group: formula-fed infants whose mothers did not smoke in pregnancy and (4) formula-fed smoking group: formula-fed infants whose mothers smoked in pregnancy.

To test whether FM and FFM differed between groups (goatmilk formula and cow-milk formula, or combined formula-fed and breastfed infants), linear regression models were fitted using a time-by-group interaction term, with time treated as categorical. An interaction term was included in the model to test whether the trajectory of body composition measures over time differed between the two groups, and estimates of the group differences were derived separately for each group and time point. A generalised estimating equation with autoregressive degree working correlation and robust variance estimation was used to account for repeated measures. Pairwise differences between groups were estimated for each time point (1, 2, 3, 4, 6 and 12 months). Both unadjusted and adjusted analyses were performed. Unadjusted analyses included only time, feeding group (cow-milk $v$. goat-milk formula or combined formulafed $v$. breastfed) and the interaction between feeding group and time. The adjusted analyses included study centre, sex, maternal smoking during pregnancy and maternal education as covariates. For the sub-group analysis by maternal smoking status, unadjusted analyses included only time, feeding by smoking group and the interaction between group and time. The adjusted analyses included study centre and maternal education.

Statistical significance was assessed at the 0.05 level unless otherwise specified. A minimal sample size of 200 (100 per group) and 788 (394 per group) is required to detect a mean difference (MD) of 200 and $100 \mathrm{~g}$ (assumed SD $500 \mathrm{~g}$ ), respectively, in FFM between the breastfed and formula-fed groups with $80 \%$ power. To detect an MD of 2 and $1 \%$ (assumed SD $3 \%$ ), respectively, in FFM\% between the groups, minimal sample sizes of 74 (37 per group) and 286 (143 per group) are required. Based on the sample size available, our study had $85 \%$ power to detect a statistically significant difference of $180 \mathrm{~g}$ in FFM (the observed MD in our study) between the formula-fed and breastfed infants at 6 months of age, and $95 \%$ power to detect $1.8 \%$ difference in FFM\% between the breastfed non-smoking and formula-fed smoking groups at 1 month of age. Analyses were carried out using Stata version 13 (Stata Corp.) or SAS version 9.3 (SAS Institute Inc.).

\section{Results}

Socio-demographic and birth anthropometric characteristics

Of the original 301 infants in the TIGGA trial (100 breastfed and 201 formula-fed), 290 (100 breastfed and 190 formula-fed) had BIS measures taken at one or more time points and were included in the present study. The remaining eleven infants did not have any BIS measures and were excluded from the present study (see online Supplementary Fig. S1, Participant flow chart). Parent and child characteristics are summarised in Table 1. Breastfed infants had a lower percentage of mothers who smoked during pregnancy, a lower maternal pre-pregnancy BMI, a higher percentage of mothers who had completed secondary education and a higher mean birth weight compared with formula-fed infants (Table 1). The birth anthropometric measures and their $z$-scores for the infants in the breastfed nonsmoking group did not differ from infants in the formula-fed non-smoking group (Table 1) but were significantly higher than the infants from the formula-fed smoking group. Infants in the formula-fed non-smoking group also had significantly higher anthropometric measures compared with the infants in the formula-fed smoking group with the exception that the differences in the head circumference and its $z$-score were not statistically significant (Table 1). There were only ten infants in the breastfed smoking group; therefore, a meaningful comparison is not possible, and subsequently, this group was not included in the sub-group analyses.

\section{Body composition of goat-milk v. cow-milk formula-fed infants}

The mean $\mathrm{FM} \%$ ranged from $16 \cdot 1$ (SD 3.1) \% at 1 month to $30 \cdot 7$ (sD $4 \cdot 0$ ) \% at 12 months in the goat-milk formula-fed infants compared with 16.8 (SD 2.7) \% and 31.4 (SD 3.2) \% in the cow-milk formula-fed infants (online Supplementary Table S1). There was no difference between the two formula-fed groups in FM (g), FFM (g), or percentage of FM and FFM, at any of the time points between 1 and 12 months of age in either the unadjusted or adjusted analyses (online Supplementary Fig. S2). There were also no interaction effects of time with body composition measurements.

\section{Body composition of breastfed $v$. formula-fed infants}

The mean FM\% ranged from 16.4 (sD 2.9) \% at 1 month to 31.0 (sD 3.6) $\%$ at 12 months in the formula-fed infants, and from 17.4 (SD 3.0 ) $\%$ to 31.2 (sD 3.5) \% in the breastfed infants (online Supplementary Table S2). In the adjusted analyses, formula-fed infants had a higher FFM at 4 months (MD $160 \mathrm{~g}, 95 \%$ CI 50.4, $269.5 \mathrm{~g}, P<0.05$ ) ) and 6 months (MD $179 \mathrm{~g}, 95 \%$ CI 41.5 , 316.9 g, $P<0.05)$. However, by 12 months, FFM between formula-fed and breastfed infants was not significantly different. There was no difference in the FFM\%, or FM or FM\% at any time points between formula-fed and breastfed infants in either the unadjusted or adjusted analyses, with the exception that formula-fed infants had a lower FM (MD $-79 \mathrm{~g}, 95 \% \mathrm{CI}-134.4$, $-23.6 \mathrm{~g}, P=0.005$ ), FM\% (MD $-1.0 \%, 95 \% \mathrm{CI}-1.7,-0.2 \%$ ) and FFM (MD $-90 \mathrm{~g}, 95 \% \mathrm{CI}-174 \cdot 7,-5 \cdot 2 \mathrm{~g}, P=0.037)$ and $\mathrm{a}$ higher FFM\% (MD 1.0\%, $95 \%$ CI 0.2, 1.7\%, $P=0.011$ ) at 1 month of age compared with the breastfed infants in the unadjusted analysis (Fig. 1 and online Supplementary Table S2). There was a significant interaction effect of time with FFM, FM\% and FFM $\%(P<0.05$ for all $)$. The MD in FFM between formula-fed and breastfed infants changed from negative (lower mean FFM in formula-fed) between 1 and 3 months to positive (higher mean 
Table 1. Demographic characteristics of participants by feeding mode and maternal smoking status (Mean values and standard deviations; numbers and percentages)

\begin{tabular}{|c|c|c|c|c|c|c|}
\hline & \multicolumn{2}{|c|}{ Feeding mode $^{*}$} & \multicolumn{4}{|c|}{ Feeding mode by maternal smoking* } \\
\hline & $\mathrm{BF}(n 100)$ & $\mathrm{FF}(n 190)$ & BFNSM $(n 90)$ & FFNSM $(n 114)$ & FFSM $(n 76)$ & BFSM $(n 10)$ \\
\hline \multicolumn{7}{|c|}{ Maternal characteristics } \\
\hline \multicolumn{7}{|c|}{ Mother completed secondary education } \\
\hline$n$ & 81 & 79 & 75 & 63 & 16 & 6 \\
\hline$\%$ & $81^{\mathrm{a}}$ & $42^{b}$ & $83^{\mathrm{a}}$ & $55^{\mathrm{b}}$ & $21^{\mathrm{c}}$ & 60 \\
\hline \multicolumn{7}{|c|}{ Maternal smoking in pregnancy } \\
\hline$n$ & 10 & 76 & 0 & 0 & 76 & 10 \\
\hline$\%$ & $10^{\mathrm{a}}$ & $40^{\mathrm{b}}$ & 0 & 0 & 100 & 100 \\
\hline \multicolumn{7}{|c|}{ Maternal age (years) } \\
\hline Mean & $30 \cdot 7^{a}$ & $28 \cdot 0^{\mathrm{b}}$ & $30 \cdot 1^{\mathrm{a}}$ & $28 \cdot 1^{\mathrm{b}}$ & $27 \cdot 8^{\mathrm{b}}$ & 28.5 \\
\hline SD & $5 \cdot 2$ & $6 \cdot 3$ & 4.9 & 5.9 & 6.9 & $7 \cdot 2$ \\
\hline \multicolumn{7}{|c|}{ Maternal pre-pregnancy BMI $\left(\mathrm{kg} / \mathrm{m}^{2}\right)$} \\
\hline Mean & $24 \cdot 6^{a}$ & $27 \cdot 1^{\mathrm{b}}$ & $24 \cdot 8^{\mathrm{a}}$ & $27 \cdot 8^{\mathrm{b}}$ & $26 \cdot 1^{a, b}$ & $22 \cdot 7$ \\
\hline SD & 4.5 & 6.9 & 4.6 & $7 \cdot 5$ & 5.8 & $3 \cdot 1$ \\
\hline \multicolumn{7}{|c|}{ Infant characteristics } \\
\hline \multicolumn{7}{|c|}{ Sex: male } \\
\hline$n$ & 43 & 86 & 42 & 52 & 34 & 1 \\
\hline$\%$ & $43^{\mathrm{a}}$ & $45^{\mathrm{a}}$ & 47 & 46 & 45 & 10 \\
\hline \multicolumn{7}{|c|}{ Birth weight (g) } \\
\hline Mean & $3563^{a}$ & $3390^{b}$ & $3579^{a}$ & $3486^{a}$ & $3245^{\mathrm{b}}$ & 3425 \\
\hline SD & 410 & 443 & 394 & 417 & 445 & 545 \\
\hline \multicolumn{7}{|c|}{ Birth length (cm) } \\
\hline Mean & $50 \cdot 2^{\mathrm{a}}$ & $49 \cdot 4^{\mathrm{b}}$ & $50 \cdot 1^{\mathrm{a}}$ & $49 \cdot 7^{\mathrm{a}}$ & $48.9^{\mathrm{b}}$ & 50.5 \\
\hline SD & $2 \cdot 0$ & $2 \cdot 1$ & $2 \cdot 0$ & $2 \cdot 0$ & $2 \cdot 2$ & $2 \cdot 2$ \\
\hline \multicolumn{7}{|c|}{ Birth head circumference $(\mathrm{cm})$} \\
\hline Mean & $35 \cdot 1^{\mathrm{a}}$ & $34.7^{\mathrm{b}}$ & $35 \cdot 2^{\mathrm{a}}$ & $34 \cdot 8^{\mathrm{a}, \mathrm{b}}$ & $34.5^{\mathrm{b}}$ & $34 \cdot 2$ \\
\hline SD & 1.2 & 1.4 & 1.2 & 1.5 & 1.4 & 1.5 \\
\hline \multicolumn{7}{|c|}{ Birth weight $z$-score } \\
\hline Mean & $0.56^{\mathrm{a}}$ & $0.19^{b}$ & $0.58^{a}$ & $0.39^{a}$ & $-0 \cdot 12^{b}$ & 0.02 \\
\hline \multirow{2}{*}{\multicolumn{7}{|c|}{ Birth length $z$-score }} \\
\hline & & & & & & \\
\hline Mean & $0.36^{a}$ & $0.04^{b}$ & 0.3 & $0.13^{a}$ & $-0.29^{b}$ & 0.59 \\
\hline SD & 1.06 & 1.11 & 1.05 & 1.05 & 1.16 & 1.09 \\
\hline \multicolumn{7}{|c|}{ Birth head circumference $z$-score } \\
\hline Mean & $0.76^{a}$ & $0.42^{\mathrm{b}}$ & $0.82^{\mathrm{a}}$ & $0.53^{a, b}$ & $0.27^{b}$ & 0.37 \\
\hline $\mathrm{SD}$ & 0.97 & $1 \cdot 15$ & 0.92 & $1 \cdot 17$ & $1 \cdot 12$ & 1.35 \\
\hline
\end{tabular}

BF, breastfed infants; FF, formula-fed infants; BFNSM, breastfed infants whose mother did not smoke in pregnancy; FFNSM, formula-fed infants whose mother did not smoke in pregnancy; FFSM, formula-fed infants whose mother smoked in pregnancy; BFSM, breastfed infants whose mother smoked in pregnancy.

a,b,c Mean values or percentages within a row with unlike superscript letters were significantly different $(P<0.05)$ based on $P$-values derived from a generalised estimating equation with robust variance estimation for continuous variables and $\chi^{2}$ test for categorical variables.

* Statistical analyses were performed to compare the characteristics between feeding groups (BF $v$. FF) and between feeding by maternal smoking subgroups (BFNM $v$. FFNSM v. FFSM) separately. Due to the small number of infants $(n 10)$ in the BFSM group, a meaningful statistical comparison is not possible and statistical comparisons between BFSM and other feeding by maternal smoking sub-groups were not performed.

FFM in formula-fed) from 4 months of age although the difference was only significant at 4 and 6 months (online Supplementary Table S2). This indicated a different body composition trajectory between the breastfed and the formula-fed infants.

Sub-group analysis of breastfed $v$. formula-fed by maternal smoking status in pregnancy showed that there were no significant differences in body composition measures including FM, FM\%, FFM and FFM\% between the breastfed non-smoking and formula-fed non-smoking groups at any of the time points. In contrast, infants in the formula-fed smoking group were lower in both FM and FFM (paralleling the lower weight and length at birth) but had a higher FFM\% and lower FM\% at 1 month compared with the other two non-smoking groups in both the unadjusted or adjusted analyses (see Fig. 2 and online Supplementary Table S3). No significant differences were observed in other time points. There was also a significant interaction effect of time with
FM\% and FFM\% ( $P<0.05$ for all) suggesting a different body composition trajectory between the smoking and non-smoking groups.

\section{Discussion}

To the best of our knowledge, ours is the largest prospective study with serial measurements in growth and body composition trajectory of breastfed $v$. formula-fed infants from birth to 12 months of age. We showed that body composition differs between breastfed and formula-fed infants in early infancy. More importantly, we demonstrated for the first time that the difference in the body composition between breastfed and formula-fed infants was only observed in formula-fed infants whose mothers smoked in pregnancy in contrast to no difference between breastfed and formula-fed infants of non-smoking 
(a)
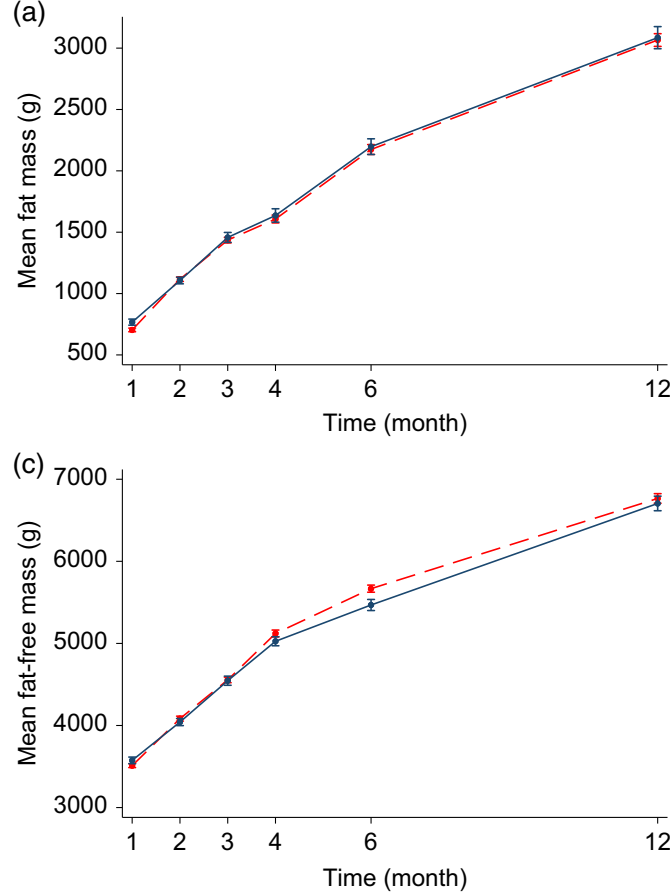

(b) 35

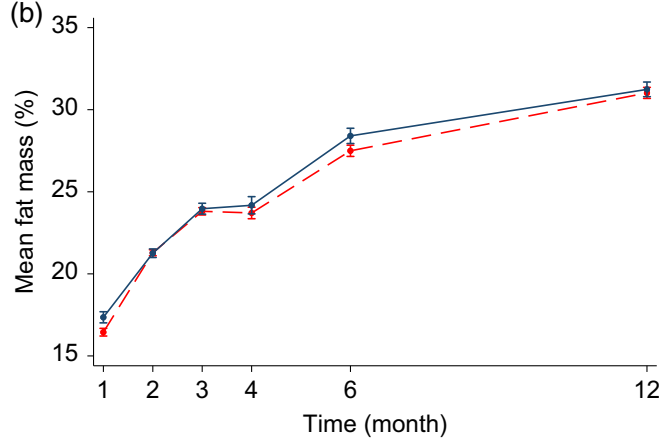

(d) 85

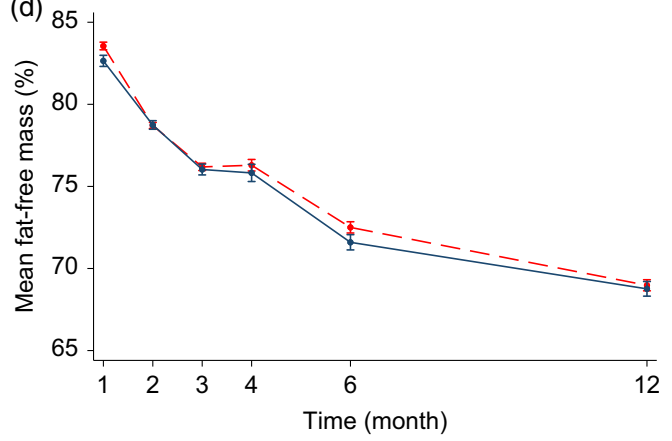

Fig. 1. Fat mass (a), fat mass percentage (b), fat-free mass (c) and fat-free mass percentage (d) of formula-fed $v$. breastfed infants in the first 12 months of age. Values are mean values with their standard errors. Error bars indicate $95 \%$ confidence intervals. A generalised estimating equation with robust variance estimation was used for statistical analysis. $\ldots \bullet$, Formula-fed $(n 190) ; \multimap$, breastfed $(n 100)$.

(a)

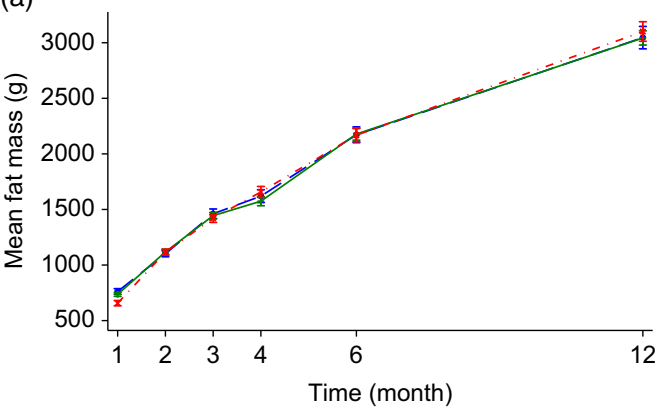

(c)

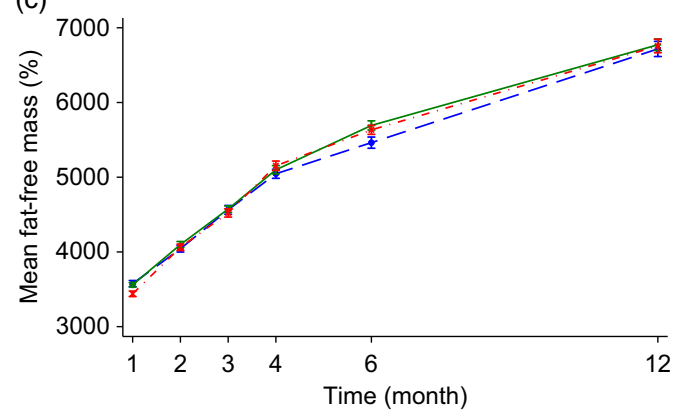

(b)

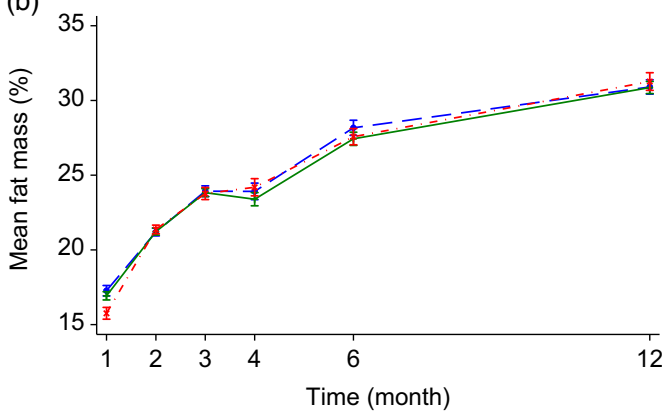

(d)

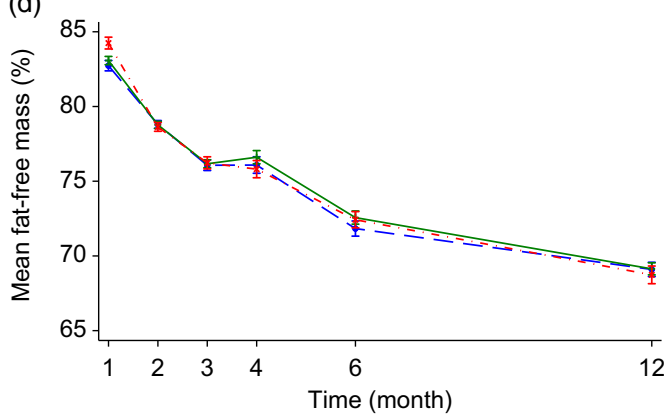

Fig. 2. Fat mass (a), fat mass percentage (b), fat-free mass (c) and fat-free mass percentage (d) of infant by feeding mode and maternal smoking status. Values are mean values with their standard errors. Error bars indicate $95 \%$ confidence intervals. A generalised estimating equation with robust variance estimation was used for statistical analysis. BF/non-smoking, breastfed infants whose mothers did not smoke in pregnancy; FF/non-smoking, formula-fed infants whose mothers did not

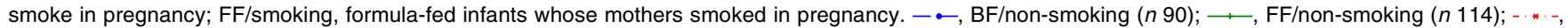
FF/smoking $(n 76)$. 
mothers, suggesting that the difference in body composition between breastfed and formula-fed infants in early infancy may potentially be attributed to maternal smoking in pregnancy irrespective of feeding mode.

Our finding that formula-fed infants had higher FFM than breastfed infants at 4 and 6 months of age is comparable with other smaller cohort studies (with twenty to seventy-six infants) that estimated body composition by total body electrical conductivity ${ }^{(21-23)}$ based on a similar principle to the one used in our study, or by other well-established methods including dual-energy X-ray absorptiometry ${ }^{(24)}$ and doubly labelled water dilution $^{(21)}$. We found that the difference in the body composition between the formula-fed and breastfed infants was no longer significant by 12 months of age, which is in contrast to a recent systematic review and meta-analysis ${ }^{(2)}$ showing higher FFM in the formula-fed infants at 12 months of age compared with the breastfed infants. However, the total number of infants included in the meta-analysis at 12 months of age was relatively small ( $n$ 201) and from three studies with different definitions of the breast-feeding group. These conflicting results are perhaps not surprising as it would be expected that any effect of feeding mode on body composition would be attenuated and less consistent in the second 6 months of the infancy, when the diet becomes more diverse with the introduction of complementary feeding as well as infant formula among some breastfed infants. Whether feeding mode in infancy has a long-term effect on the growth of children or risk of obesity later in life remains uncertain because of conflicting results from epidemiological studies. However, evidence from limited longitudinal studies demonstrated that the difference in body composition between formula-fed and breastfed infants in early infancy did not persist into the second year of life ${ }^{(22)}$. This suggests that the association between breast-feeding and the lower risk of obesity later in life may be at least in part due to other social and environmental factors, such as maternal smoking and other lifestyle practices, associated with the choice to breastfeed rather than breastfeeding itself.

There is consistent evidence demonstrating the link between maternal smoking in pregnancy and fetal growth restriction. Infants born to mothers who smoked in pregnancy are generally smaller at birth as indicated by lower mean birth weight, length and head circumference compared with infants whose mothers did not smoke in pregnancy ${ }^{(10,25)}$. Growth patterns and body composition have also been shown to differ between infants of smoking mothers and non-smoking mothers ${ }^{(12,26)}$. It is also well documented that women who chose to formula feed their infants have a significantly higher rate of smoking in pregnancy compared with those who chose to breastfeed ${ }^{(3,17,27)}$. Despite that, none of the studies comparing growth and body composition trajectory of breastfed and formula-fed infants have conducted the comparison in relation to maternal smoking status in pregnancy. Our study is the first to show no difference in the anthropometric measures (including body composition) between breastfed and formula-fed infants from non-smoking mothers. However, anthropometric measures of infants of non-smoking mothers (regardless of the feeding mode) differed from formula-fed infants whose mothers smoked in pregnancy, with the latter being smaller at birth and leaner at 1 month. Our finding suggested that the difference observed in the body composition between formula-fed and breastfed infants in our study in early infancy may potentially be attributed to maternal smoking in pregnancy irrespective of feeding mode. The underlying mechanisms for the association between maternal smoking in pregnancy and the growth and body composition of offspring are not fully understood. Several possible explanations have been suggested, including increased energy expenditure ${ }^{(28)}$, maternal malnutrition, poor weight gain in pregnancy, impaired placenta function and possible direct effect of smoking on metabolic alterations in both mothers and fetus ${ }^{(29,30)}$. Growth patterns of formula-fed infants are often viewed as sub-optimal because they deviate from the WHO growth standard, which was developed based on growth data of healthy breastfed infants whose mothers did not smoke during pregnancy ${ }^{(31)}$. However, there is a disproportionally high rate of maternal smoking in pregnancy among formula-fed infants, and the different growth patterns between formula-fed and breastfed infants should not be simply attributed to the compositional differences between breast milk and infant formula as maternal smoking in pregnancy is an important factor influencing the growth of infants.

We previously showed no difference in growth (measured by weight, length and head circumference) between infants fed goatmilk infant formula and those fed a conventional cow-milk infant formula without milk fat in a randomised controlled trial ${ }^{(17)}$. In the present study, we demonstrated that the body composition of these two groups of infants was also not different. This further supports the nutritional adequacy of goat-milk infant formula in comparison with the traditional cow-milk infant formula.

The key strengths of our study are that we tracked the body composition with serial measurements from birth to 12 months of age, which allows longitudinal comparison in body composition trajectory between the groups, and assessment of body composition was conducted blinded to maternal smoking status in pregnancy in the whole cohort. Our study has some limitations. We did not assess the number of daily cigarettes smoked or second-hand smoking in pregnancy or adjust for the age when solids were introduced, which could be potential confounders of the body composition in infancy. Our sample size afforded adequate power to detect the observed difference in FFM between the formula-fed and breastfed infants at 4 and 6 months of age and the FFM and FFM\% between the breastfed nonsmoking and formula-fed smoking groups at 1 month of age. A larger sample size is required to detect a smaller difference in body composition between groups and to account for multiple comparisons between feeding groups by maternal smoking status in pregnancy. Our results should be interpreted in the context of limited statistical power. Whether body composition trajectory differs between breastfed and formula-fed infants whose mothers smoked in pregnancy is unknown as we were unable to compare the groups due to inadequate number of mothers who smoked in pregnancy and breastfed their infants in our study.

In summary, our results suggest that maternal smoking in pregnancy influences the body composition of infants independent of the feeding mode in infancy. Educating women on the impact of maternal smoking on infant growth and providing support for the cessation of smoking should be an integral part of any public health campaign and antenatal care to optimise the 
growth and long-term health of children. Further research with adequately powered prospective studies, specifically designed to examine the relationship between maternal smoking in pregnancy, feeding mode and the body composition of infants, is warranted to better understand the relative impact of maternal smoking in pregnancy, feeding practice in early life and growth trajectory of infants.

\section{Acknowledgements}

The authors thank the families who participated, the medical, nursing and research staff in each participating centre, the staff of the Child Nutrition Research Centre, South Australian Health and Medical Research Institute and the staff of the Data Management and Analysis Centre, The University of Adelaide.

The present study utilised the data collected in a randomised controlled trial that was funded by the Dairy Goat Co-operative (N.Z.) Ltd, New Zealand. The study design, statistical analysis, interpretation of findings and the preparation of the manuscript were conducted independently of the Dairy Goat Co-operative (NZ) Ltd. Statistical analysis was supported by the Centre of Research Excellence funded by the National Health and Medical Research Council (grant no. 1035530). M. M. and R. A. G. were supported by the National Health and Medical Research Council Research Fellowship (Makrides' Fellowship ID: APP106174 and Gibson's Fellowship ID: APP 1046207). C. T. C. was supported by a Women's and Children's Hospital MS McLeod Postdoctoral Research Fellowship.

Study design: S. J. Z., M. M., R. A. G. and C. T. C. Study management: S. J. Z. and M. M. Supervising body composition assessment: C. T. C. Statistical analyses: K. H. Drafting the manuscript: S. J. Z. drafted the manuscript with contributions from K. H. Primary responsibility for the final content: S. J. Z. and M. M. All authors read and approved the final manuscript.

M. M. serves on scientific advisory boards for Nestle and Fonterra. R. A. G. serves on scientific advisory board for Fonterra. Associated honoraria for M. M. and R. A. G. are paid to their institutions to support conference travel and continuing education for post-graduate students and early career researchers. No other conflicts of interest were reported.

\section{Supplementary material}

For supplementary materials referred to in this article, please visit https://doi.org/10.1017/S0007114519002848

\section{References}

1. Dewey KG (1998) Growth characteristics of breast-fed compared to formula-fed infants. Biol Neonate 74, 94-105.

2. Gale C, Logan KM, Santhakumaran S, et al. (2012) Effect of breastfeeding compared with formula feeding on infant body composition: a systematic review and meta-analysis. $\mathrm{Am}$ J Clin Nutr 95, 656-669.

3. Koletzko B, von Kries R, Closa R, et al. (2009) Lower protein in infant formula is associated with lower weight up to age $2 \mathrm{y}$ : a randomized clinical trial. Am J Clin Nutr 89, 1836-1845.
4. Dewey KG (1998) Growth patterns of breastfed infants and the current status of growth charts for infants.J Hum Lact 14, 89-92.

5. de Beer M, Vrijkotte TG, Fall CH, et al. (2015) Associations of infant feeding and timing of linear growth and relative weight gain during early life with childhood body composition. Int J Obes (2005) 39, 586-592.

6. Ejlerskov KT, Christensen LB, Ritz C, et al. (2015) The impact of early growth patterns and infant feeding on body composition at 3 years of age. Br J Nutr 114, 316-327.

7. Robinson SM, Marriott LD, Crozier SR, et al. (2009) Variations in infant feeding practice are associated with body composition in childhood: a prospective cohort study. J Clin Endocrinol Metab 94, 2799-2805.

8. Owen CG, Martin RM, Whincup PH, et al. (2005) The effect of breastfeeding on mean body mass index throughout life: a quantitative review of published and unpublished observational evidence. Am J Clin Nutr 82, 1298-1307.

9. Harrod CS, Reynolds RM, Chasan-Taber L, et al. (2014) Quantity and timing of maternal prenatal smoking on neonatal body composition: the Healthy Start study. J Pediatr 165, 707-712.

10. Samper MP, Jimenez-Muro A, Nerin I, et al. (2012) Maternal active smoking and newborn body composition. Early Hum Dev 88, 141-145.

11. Harrison GG, Branson RS \& Vaucher YE (1983) Association of maternal smoking with body composition of the newborn. Am J Clin Nutr 38, 757-762.

12. Harrod CS, Fingerlin TE, Chasan-Taber L, et al. (2015) Exposure to prenatal smoking and early-life body composition: the healthy start study. Obesity (Silver Spring) 23, 234-241.

13. Spady DW, Atrens MA, Szymanski WA (1986) Effects of mother's smoking on their infants' body composition as determined by total body potassium. Pediatr Res 20, 716-719.

14. Cameron N, Pettifor J, De Wet T, et al. (2003) The relationship of rapid weight gain in infancy to obesity and skeletal maturity in childhood. Obes Res 11, 457-460.

15. Ong KK, Ahmed ML, Emmett PM, et al. (2000) Association between postnatal catch-up growth and obesity in childhood: prospective cohort study. BMJ 320, 967-971.

16. Hawke K, Louise J, Collins C, et al. (2017) Growth patterns during the first 12 months of life: post-hoc analysis for South Australian Aboriginal and Caucasian infants in a randomised controlled trial of formula feeding. Asia Pac J Clin Nutr 26, 464-470.

17. Zhou SJ, Sullivan T, Gibson RA, et al. (2014) Nutritional adequacy of goat milk infant formulas for term infants: a double-blind randomised controlled trial. Br J Nutr 111, 1641-1651.

18. WHO Multicentre Growth Reference Study Group (2006) WHO Child Growth Standards based on length/height, weight and age. Acta Paediatr Suppl 450, 76-85.

19. Lingwood BE, Storm van Leeuwen AM, Carberry AE, et al. (2012) Prediction of fat-free mass and percentage of body fat in neonates using bioelectrical impedance analysis and anthropometric measures: validation against the PEA POD. Br J Nutr 107, 1545-1552.

20. Collins CT, Reid J, Makrides M, et al. (2013) Prediction of body water compartments in preterm infants by bioelectrical impedance spectroscopy. Eur J Clin Nutr 67, Suppl. 1, S47-S53.

21. Butte NF, Wong WW, Fiorotto M, et al. (1995) Influence of early feeding mode on body composition of infants. Biol Neonate 67, 414-424.

22. Butte NF, Wong WW, Hopkinson JM, et al. (2000) Infant feeding mode affects early growth and body composition. Pediatrics 106, 1355-1366.

23. de Bruin NC, Degenhart HJ, Gal S, et al. (1998) Energy utilization and growth in breast-fed and formula-fed infants measured 
prospectively during the first year of life. Am J Clin Nutr $\mathbf{6 7}$, 885-896.

24. Bell KA, Wagner CL, Feldman HA, et al. (2017) Associations of infant feeding with trajectories of body composition and growth. Am J Clin Nutr 106, 491-498.

25. Zaren B, Lindmark G \& Gebre-Medhin M (1996) Maternal smoking and body composition of the newborn. Acta Paediatr 85, 213-219.

26. Harvey NC, Poole JR, Javaid MK, et al. (2007) Parental determinants of neonatal body composition. J Clin Endocrinol Metab 92, 523-526.

27. Hawkes JS, Gibson RA, Roberton D, et al. (2006) Effect of dietary nucleotide supplementation on growth and immune function in term infants: a randomized controlled trial. Eur J Clin Nutr 60, 254-264.
28. Hofstetter A, Schutz Y, Jequier E, et al. (1986) Increased 24-hour energy expenditure in cigarette smokers. $N$ Engl $J$ Med 314, 79-82.

29. Li MD, Parker SL \& Kane JK (2000) Regulation of feeding-associated peptides and receptors by nicotine. Mol Neurobiol 22, 143-165.

30. Pollack RN \& Divon MY (1992) Intrauterine growth retardation: definition, classification, and etiology. Clin Obstet Gynecol 35, 99-107.

31. Turck D, Michaelsen KF, Shamir R, et al. (2013) World Health Organization 2006 child growth standards and 2007 growth reference charts: a discussion paper by the committee on Nutrition of the European Society for Pediatric Gastroenterology, Hepatology, and Nutrition. I Pediatr Gastroenterol Nutr 57, 258-264. 